\title{
Improving support for sexual trauma
}

\author{
Kamran Abbasi executive editor \\ The BMJ
}

What are the long term health needs of women with a history of sexual trauma? This question isn't well covered in guidelines, since they tend to focus on acute treatment after a sexual attack. Over a lifetime, around $30 \%$ of the world's women will report intimate partner violence, whether physical, sexual, or both, and $7 \%$ will face non-partner sexual violence.

You might be told about a sexual assault months or years after it occurred. What to do? In a clinical update, Veronica Ades and colleagues provide practical advice on how to help women even though the traumatic event may be some time in the past (doi:10.1136/bmj.15825). They also explain the long term effects on mental and physical health. Among forms of trauma, sexual violence is one of the strongest predictors of post-traumatic stress disorder, for example. Every little bit of support helps.

Unlike sexual assault, hypertension is covered by every possible guideline. Guidance published in August by the UK National Institute for Health and Clinical Excellence is now summarised in The BMJ (doi:10.1136/bmj.15310). We've made clear what's new, such as taking into account patients' preferences,

maintaining blood pressure below target rather than aiming for it, and a new approach to managing people with asymptomatic severe hypertension. We've also produced an infographic on hypertension in adults to help you follow the latest guidance (bmj.com/content/367/bmj.15310/infographic). Meanwhile, a separate study indicates that taking antihypertensives at bedtime instead of in the morning improves blood pressure control (doi:10.1136/bmj.16173).

Joshua Petimar and colleagues tested a new approach to reducing obesity by calorie labelling of menus in a large restaurant franchise (doi:10.1136/bmj.15837). They noticed an immediate effect on calorie content of purchased food, but the effect diminished over one year. Editorialists Adam Briggs and Asha Kaur are undeterred (doi:10.1136/bmj.16119). Calorie labelling has a role, they argue, in a multifaceted approach to tackle the complex challenge of obesity, whereby "small changes to calorie intake can have meaningful effects at the population level."

In 2016 the World Bank devised a financing scheme-a big change - to fund a swift response to potential pandemic outbreaks, such as the Ebola epidemic. The Pandemic Emergency Financing Facility was welcomed as an innovative solution, but it is yet to make a payout. Indeed, the rules for releasing funds seem so stringent that a payout would have been triggered only twice since 2006 (doi:10.1136/bmj.15719). Alarmingly, the scheme has paid $\$ 114.5 \mathrm{~m}$ to investors. Big isn't necessarily better. 